\title{
Images plurielles de l'identité de genre chez Hugues Corriveau et Normand de Bellefeuille à La Nouvelle Barre du jour
}

\author{
Élyse Guay \\ Université du Québec à Montréal
}

En 1985, dans la revue Voix et images, l'écrivaine et critique Gabrielle Frémont s'interroge :

[...] la grande victoire de l'écriture féministe de ces dernières années ne serait[elle] pas d'avoir sensibilisé les hommes (et quelques femmes aussi) aux conditions précaires et souvent inacceptables faites aux femmes dans tous les domaines - social, sexuel, politique et affectif[?] En tout cas, une chose est certaine, du moins à la NBJ [Nouvelle Barre du jour], les poètes et critiques masculins n'écrivent plus de la même façon au sujet des femmes. On découvre enfin chez eux - et cela paraît relativement nouveau - une oreille attentive à la parole de l'autre, un respect aussi de ce qu'elle est et de ce qu'elle dit désormais vouloir être (p. 136). 
Partant de ce commentaire de la directrice du dossier-manifeste FÉMINaire d'Études littéraires en 1979, je me propose d'interroger la poésie d'inspiration féministe, qui a été principalement élaborée au Québec au sein de la revue la Nouvelle Barre du jour1.

La relecture de ce corpus tend à faire apparaître diverses poétiques qui émanent du formalisme littéraire des années 1970. En effet, deux signatures que l'on associe plus souvent aux herbes rouges (1968-), soit Normand de Bellefeuille et Hugues Corriveau, apparaissent avec récurrence, entre 1975 et 1980, au sommaire de la $B J / N B J$. Le second arrive en tête des collaborateurs et collaboratrices de la revue, aux côtés de Michel Beaulieu, France Théorêt et Nicole Brossard. À la lumière de ce constat, il s'agira d'observer le discours d'un certain nombre d'auteurs masculins et les procédés textuels utilisés, en postulant qu'ils participent, d'une manière originale, aux représentations renouvelées de l'identité sexuelle et du genre (gender). Je développe l'hypothèse que la refonte de la revue, en 1977, indique un moment de glissement, une sorte de passage entre deux périodes ou régimes historiques, qui annonce le croisement d'un discours féministe radical avec une pluralité de voix. Retracer les permutations de l'avant-garde poétique québécoise dans son tournant postmoderne, objectif qui s'inscrit dans une recherche plus vaste sur les périodiques (Guay, 2015), me permettra d'analyser l'apport des écrivains masculins que pointe Gabrielle Frémont durant une période où la pensée féministe évolue rapidement. Je ciblerai les stratégies qu'ils ont adoptées afin de mettre en scène une certaine hybridité des pratiques à la $N B J$.

\footnotetext{
${ }^{1}$ Les prochaines références à la Barre du jour (1965-1977) ou à la Nouvelle Barre du jour (1977-1990) seront indiquées avec les mentions $B J$ ou $N B J$.
} 


\section{Entre rupture et tradition : les linéaments du formalisme}

Si Pierre Nepveu (1985), Jean Larose (1987), Jacques Pelletier (1986) et Pierre Milot (1988) ont désigné la $B J$ comme le lieu de rencontre et d'élaboration de «l'avantgarde formaliste », il faut toutefois rappeler qu'elle y a pris place en s'opposant progressivement à une certaine politisation de la littérature. En effet, la première mouture de la revue se place « sous le signe paradoxal de la rupture et la continuité » (Biron, Dumont, Nardout-Lafarge, 2010, p. 490). Fondée en 1965, la $B J$ marque à la fois une distance à l'égard de la poésie du pays et un parti pris pour la littérature qui passe par la tradition. Cela se traduit avec la section « Les inédits » où l'équipe forge un corpus canadien-français lié à l’École littéraire de Montréal (Dion, 1994, p. 215) et s'inscrit en filiation avec ces auteurs du XIXe siècle. La revue se démarque de la poésie du pays par la reconnaissance de figures qui incarnent l'autonomie du littéraire ou le motif de la révolution², ce qui renforce sa position d'avant-garde dans la sphère restreinte du champ littéraire.

En filigrane de ces numéros «hommages », l'équipe de la BJ en 1968 (Nicole Brossard, Jan Stafford, Roger Soublière et Marcel Saint-Pierre) développe un formalisme littéraire et théorique inspiré de la revue française Tel Quel dans sa phase structuraliste. Loin d'être apolitique, le comité de rédaction, que Michel Beaulieu a

\footnotetext{
2 Roland Giguère (n $\left.{ }^{\text {os }} 11-12-13,1968\right)$, les Automatistes (nos 17-18-19-20, 1969), Saint-Denys Garneau (sept.-oct. 1969), Gaston Miron à la suite d'Octobre 1970 (1970) et Parti pris (n ${ }^{\text {os }} 31-32,1972$ ) font tous l'objet d'imposants dossiers. Concernant les deux derniers numéros, l'équipe y opère une relecture de la poésie du pays qui met en relief l'idée de révolution. L'intérêt pour ce motif poétique sert de socle au formalisme littéraire en émergence, bien qu'il se situe en marge des courants politiques, et se traduira par des textes violemment déconstruits et subversifs.
} 
rejoint (1970-1973) ${ }^{3}$, situe son engagement dans le langage, contre les formes de doxa et de pouvoir. L’autoréflexivité, la syntaxe éclatée, l'intertextualité, les jeux sur le signifiant, le graphisme et la typographie sont autant de procédés qui caractérisent leur démarche formelle. Le collectif de la revue, ainsi que d'autres écrivaines et écrivains rassemblés autour des herbes rouges, rejettent avec vigueur la poésie figurative et lyrique associée à l'Hexagone et à Liberté (1959-).

\section{Une poésie d'inspiration féministe à la $\mathrm{BJ}$}

Le numéro Transgression, paru à l'automne 1973, montre que la BJ est devenue le catalyseur de l'avant-garde formaliste ralliant aux côtés de Nicole Brossard, les contre-culturels d'Hobo-Québec (Raôul Duguay, Paul Chamberland, Lucien Francoeur,

Patrick Straram et André Beaudet) et François Charron, qui poursuit son travail théorique inspiré de la sémiologie française et du structuro-marxisme. À ce « courant » formaliste s'ajoute un élan sémiologique qui interroge le rôle de l'art et de la littérature au sein de la société, débat déjà lancé par Charron à la revue Stratégie (1972-77), fondée un an auparavant. Qui plus est, dans ce numéro ayant pour thème la transgression, en plus de la dimension ludique et transgressive insufflée par les

\footnotetext{
3 Figure importante de la nouvelle génération de poètes et fondateur des Éditions l'Estérel (1965), Michel Beaulieu a collaboré à la $B J$ dès 1968. Un an auparavant, une querelle divise les membres du nouveau comité de rédaction de la revue (Beaudry, 1998, p. 10) et provoque son départ. Par la suite, Beaulieu lance, notamment avec Raôul Duguay, la revue Quoi (1967) dont le manifeste du second et ultime numéro revendique une créativité qui passe par l'invention formelle: "[1]a politique nous intéresse, mais nous sommes d'abord des écrivains. Et nous ne croyons pas que l'écriture doive s'asservir à la politique. L'écriture est donc pour nous un geste sans signification autre qu'en lui-même. Pas de rhétorique, pas de thèse à défendre, que l'écriture. Et l'invention. [...] Nous avertissons la critique : elle se fourvoiera dès qu'elle considérera l'écriture en dehors de sa structure formelle, de son invention » (Y. M., 1967, p. 3-6).
} 
explorations textuelles des poètes de la contre-culture, Brossard pose la question des

femmes.

Intitulé « Vaseline », le texte de Brossard esquisse la fonction de l'énergie qu'a ciblée Pierre Nepveu : transformer l'exclusion symbolique des femmes dans le langage en puissance créatrice, en écriture du désir (1988, p. 150). L'écriture chez Brossard invite à la dérive et au mouvement pour créer des formes nouvelles, notamment au moyen de la métaphore du «corps-texte » qu'elle emploie aux côtés des écrivaines féministes durant les années 19704 . Ce jeu d'amalgame entre en concurrence avec le nouage «sexe-texte» mis de l'avant chez les poètes des herbes rouges, qui publient aussi dans les revues contre-culturelles Cul Q (1973-76)5 et Hobo-Québec (1973-81) ${ }^{6}$.

En 1973, Brossard écrit, quant à elle :

[u]ne grammaire ayant pour règle : le masculin l'emporte sur le féminin doit être transgressée, susciter quelques manœuvres de fond, de lames, de plaisir... d'humour (ce petit e muet (féminin) qu'on ajoute à la forme du masculin), epsilon ironique qui modifie insidieusement le ventre de la femme-mère castratrice et du complexe qui s'en suit (p. 14).

\footnotetext{
4 À la même époque, la métaphore du «corps-texte» n'est pas seulement l'apanage des écrivaines québécoises qui l'ont développée conjointement avec leurs consœurs du mouvement féministe en France (Hélène Cixous, Annie Leclerc). À la revue Dérives (1975-1987), la poésie de Jean Jonassaint lance un appel à la solidarité mixte qui s'appuie notamment sur des représentations hybrides du corps et de l'identité de genre afin d'intégrer la problématique de l'appartenance ethnoculturelle au champ littéraire, esquissant certains des principaux enjeux de l'écriture migrante (Guay, 2015).

${ }^{5}$ Signé Claude Beausoleil et André Roy, le manifeste du numéro 4-5 de Cul $Q$, dédié aux fictions théoriques, est clair : «[d]'où l'écriture comme mise au jeu pré-coïtale dont le résultat de la prégnance serait moins le livre qu'une articulation fixant/figeant, gelant/irrémédiablement les désirs. D'où la lecture en copie conforme à l'acte sexuel. D'où l'écriture comme lit de procuration érotique signalant le recommencement de l'accouplement sexuel/textuel » (1974, p. 39).

${ }^{6}$ L'opposition entre la $B J$ et les herbes rouges doit être nuancée. La revue de Brossard et la maison d'édition des frères Hébert ne forment pas deux groupes distincts. Tous deux s'échangent la majorité de leurs collaborateurs. De façon schématique, nous pouvons observer que les textes les plus corrosifs, vulgaires ou misogynes (plus près de la contre-culture) ne sont pas publiés à la $B J$ et que le féminisme trouve un lieu d'élaboration grandissant aux herbes rouges, avec France Théoret, Madeleine Gagnon et Josée Yvon, dont la posture est parfois controversée.
} 
On voit ici les germes d'un investissement féministe et militant qui va se déployer dans maints lieux et revues (Bergeron, 2013; Boisclair, 2004). N'empêche qu'en 1973, l'écriture des femmes apparaît comme une nouvelle orientation poétique dans la sphère de production restreinte du champ littéraire québécois. Or, il faut signaler qu'à l'opposé des animatrices de Québécoises deboutte! (1971-74) et de Têtes de pioche (1976), l'équipe de la $B J$ ne se crée pas d'identité collective forte. À la manière d'un lieu de ralliement, les dossiers qui sollicitent la collaboration exclusive d'écrivaines - Femme et langage $\left(\mathrm{n}^{0} 50,1975\right)$ et Le corps, les mots, l'imaginaire (n $\left.{ }^{\text {os }} 56-57,1977\right)$ - font de leur geste d'écriture un acte politique. Si la série de numéros féministes se poursuit jusqu'à l'orée de la décennie 1990 (Forsyth, 1983, p. 175), plusieurs auteurs masculins, parmi les livraisons subséquentes, témoignent d'un travail intellectuel et littéraire en faveur de ce mouvement. En cela, je postule qu'à la fin des années 1970, la $N B J$ a mis en place un réseau mixte, qui lui confère un caractère hétérogène et assure son renouvellement vers un métaféminisme, critique et ouvert (Boisclair, 2004).

\section{Poétiques et images collectives à La Nouvelle Barre du jour}

De par sa nature plurielle, la revue est un lieu d'énonciation particulier, « forum à voix multiples et simultanées » (1990, p. 169), comme le souligne la sociologue Andrée Fortin. C'est également un lieu de sociabilité, un «point de rencontre d'itinéraires individuels » (Pluet-Despatin, 1992, p.126) en une seule et même publication, qui peut faire apparaître des voix discordantes. Ce sont plutôt des voix solidaires auxquelles je voudrais faire écho grâce à une courte étude des poétiques et des images 
collectives qui se déploient au sein des textes d'un groupe d'écrivains masculins à la NBJ.

Le corpus se compose d'une quinzaine de textes, parus entre 1978 et 1982, et unit Hugues Corriveau, Normand de Bellefeuille, Joseph Bonenfant, Claude Beausoleil et André Beaudet. Les contributions de Michel Gay et de Jean-Yves Colette, respectivement codirecteurs de la NBJ (1977-1981), ont été mises de côté. Leurs représentations de la femme auraient pu servir de repoussoir : les figures d'amants traînent parfois des relents hétéronormatifs ${ }^{7}$, de «[p]etites misères du masculin singulier» (1981), pour reprendre l'expression de Pierre Nepveu à propos de la question de la masculinité face au mouvement des femmes ${ }^{8}$. Décrire ces représentations qui posent la femme en tant qu'objet et non comme sujet aurait donné lieu à une comparaison simpliste, voire antagonique, des discours, notamment en raison du manque d'espace pour mener à bien une analyse de leurs principaux topoï et figures. Parmi l'ensemble des textes masculins choisis, deux genres se sont rapidement imposés : essais et proses poétiques. Préférant cibler la genèse d'un discours solidaire au mouvement féministe, j'ai aussi retenu une «fiction théorique »,

\footnotetext{
7 « Ton corps nu/tout encore retenu/et partant de tes joues/la pâleur dont le secret/te fait plus belle encore/ta bouche brève au goût/[...]/rappelle-moi aussi/ta manière de nous lisser les cheveux/et le calme délire de tes yeux pâles/ou cet endroit/qui cache l'extrême-intérieur/ton ventre où je n'ai pas encore semé/[...]/violer/ta main à ma convenance/et ta gorge de mon souffle/et de ma chair outragée/outrager ta voix à mon gré/que tu ne sois plus/que ces liquides mi-salés/qui me peuplent la bouche» (Colette, novembre 1977, p. 24, 26, 28). À titre d'exemple, un extrait de Michel Gay : «où s'attarder devient de plus en plus difficile à décider et avant qu'une seule cicatrice n'ait pu refermer la fente, qu'il sache alors où, il s'en tirera de plus belle dans la cervelle. ${ }^{\circ}$ jusque-là gesticule continu, il la fera sauter sur ses genoux comme un joujou » (Gay, janvier 1978, p. 49).

8 Dans un compte rendu paru dans Lettres québécoises, Nepveu recense quatre numéros des herbes rouges. Si Marcel Labine, André Roy et Pierre Monette sont écorchés, il note que "Corriveau aborde [dans Du masculin au singulier] avec courage et sans frivolité la question on ne peut plus actuelle de la masculinité face au mouvement des femmes » (Nepveu, 1981, p. 30).
} 
genre littéraire hybride caractéristique des années 1970, mêlant l’idéologique à la poésie au moyen d'une esthétique du fragment.

Le cas de Normand de Bellefeuille, auteur associé aux herbes rouges, remet en question les représentations de l'avant-garde féministe, conçue en tant que mouvance littéraire homogène et non-mixte. Ses contributions disent le désir de participer à cette écriture en ébullition, liée de près au mouvement politique et social de revendication de l'égalité. Septembre 1977 marque l'acte de naissance de $L a$ Nouvelle Barre du jour et la création des sections «Essai » et «Commentaires » de la revue. Le premier article à l'étude est un essai intitulé «Le corps mineur ou l'impossible lyrisme », où Normand de Bellefeuille prend position contre des critiques du milieu littéraire québécois. Il déplore d'entrée de jeu :

[o]n en est là, à la mauvaise conscience, aux grandes mesures, aux nuances, celles entre poème et texte, entre poète et travailleurs de l'écriture (titre éminemment questionnable.) [...] Quand de mon écriture on a dit qu'elle était féminine, c'était paraît-il à cause du lyrisme qu'elle proposait, quand on a sousentendu qu'elle était réactionnaire et pourquoi pas un peu arriérée c'était là encore dû à ses lyriques tendances, quand on en a vanté la nouveauté et l'audace c'était bien sûr redevable au "nouveau» lyrisme qu'elle offrait. Décidément bien utile le lyrisme. Le temps n'est pas au lyrisme, on en est donc aux accusations (Bellefeuille, septembre 1977, p. 84).

Opposé avec vigueur au lyrisme qu’on lui accole9, il dénonce au passage la propension à associer certains traits de l'écriture au sexe biologique. Bellefeuille se distancie ensuite de la génération qui l'a précédé en plaçant en exergue une citation de

\footnotetext{
${ }^{9}$ La querelle en cours aboutit, selon plusieurs historiens de la littérature québécoise dont Bernard Andrès (1986, p. 105-116), avec le dossier «Intellectuel/le en 1984? » (La Nouvelle Barre du jour, no 130-131, octobre 1983) où Normand de Bellefeuille, Hugues Corriveau et Louise Dupré font la promotion du féminisme et d'un certain progressisme à la $N B J$ en opposition avec le " retour au sacré » revendiqué par François Charron et André Beaudet ("Qui a peur de l'écrivain? », Herbes rouges, no 123124, 1984).
} 
Terre Québec et commente : "[l]e temps manque (relire "Entre nous le pays" de Chamberland) et presque pour s'en excuser cette poésie d'hommes féminisera l'espace, le pays, l'aurore et jusqu'à la révolution. On parlera alors de "lyrisme nationaliste"» (septembre 1977, p.85), courant auquel il ne s'identifie pas. Rapidement, Bellefeuille fait intervenir les paroles de Claude Beausoleil dans la revue Hobo-Québec, affirmant que «le Texte a remplacé le Pays » (Beausoleil, 1977, p. 19) et précise que la métaphore du «corps-texte, l'écriture désirante » à la manière de Brossard, tente de renouveler la poésie québécoise (Bellefeuille, septembre 1977, p. 87), sans trop de succès. Autrement dit, Bellefeuille adresse d'abord de virulentes critiques à la génération de l'Hexagone et aux animateurs de Parti pris pour se distancier de leur lyrisme et des inclinaisons sexuées de leurs principaux motifs. Dans un second temps, il réfère à ses collègues écrivains et poètes d'avant-garde (Nicole Brossard, Roger des Roches, etc.), dont les pratiques, centrées sur les notions d'écriture et de texte, tournent à vide, s'épuisent.

Sa relecture de «deux moments stratégiques de la poésie québécoise» (Bellefeuille, septembre 1977, p. 86) lui permet alors d'exprimer son rapport à l'écriture. L'opposition engagement/subversion, tension qui surgit à nouveau par les récents textes qu'il convoque, signale un certain retour à la lisibilité. Bellefeuille multiplie en effet les épigraphes tirées des productions de Marcel Labine, François Charron et Madeleine Gagnon, ajoutant : « [l]e corps politique, le corps social, le corps familial, le corps conjugal seront patiemment investis par le discours critique de ceux et celles pour qui le texte se conçoit dorénavant non comme une fin mais comme un 
moyen, un constat, un attentat» (Bellefeuille, septembre 1977, p. 88). L'écrivain souhaite ainsi dépasser la simple «transformation du matériau linguistique » (Bellefeuille, septembre 1977, p. 89) afin d'interroger les structures qui produisent des discours aliénants sur la famille, le couple, le système politique, etc.

Bellefeuille poursuit dans la même veine au numéro 60. Son compte rendu « La gageure du lisible », ouvrant la section «Commentaires », décrit à nouveau le climat de méfiance qui pèse sur le milieu littéraire : «[p]arler du texte de l'ami ne devrait rien avoir de dérangeant, moins encore de restreignant. Pourtant ça se surveille terriblement, ça s'empêche même et ça hésite. Ça sait bien que ce texte, alors qu'il n’était que parole, plaisait déjà, marquait ma propre écriture de ses mots, de son agitation, de sa lisibilité »(Bellefeuille, novembre 1977, p.70). Il encense ici Serge Gauthier, auteur maison des herbes rouges, chez qui la parole devient « revendicatrice, militante » et fait surtout entendre « la parole bafouée de la blanchisseuse, de la secrétaire, de la couturière, de la femme d'intérieur » (Bellefeuille, novembre 1977, p. 71).

Comme le souligne Louise Dupré dans son essai Stratégies du vertige, durant la deuxième moitié des années 1970, un retour à la lisibilité se fait sentir et déborde des milieux féministes (1989, p.17-18). D’autres revues poétiques témoignent de l'épuisement des «machines» formalistes et des avant-gardes: Estuaire (1976-), Moebius (1977-) et Lèvres urbaines (1983-92) renouent avec une poésie 
d'«émotion »10. À Estuaire, Jean Royer et Pierre Morency valorisent une «pratique incarnée de la poésie » (mai, août 1977, p. 262), un mélange entre lyrisme exacerbé et limpidité, différent de ce que proposent Bellefeuille et Hugues Corriveau. Poète aux herbes rouges, Corriveau milite dans les pages de la $N B J$ pour une poésie qui laisse place à la subjectivité et au lisible, pour une écriture qui « dit» la femme. Selon les recherches effectuées sur les collaboratrices et collaborateurs de la $B J / N B J$, entre 1975 et 1980, Corriveau est le plus productif avec 17 textes en 50 livraisons.

En 1978, Hugues Corriveau recense un récent essai d'esthétique de Gilbert Lascault (1977). La démarche du critique d'art, basée sur l'étude des représentations de la femme, lui permet de mettre en relief «le risque énorme d'offrir aujourd'hui un discours d'homme au sujet de la femme » (Corriveau, juin 1978, p. 71), une mise en garde similaire à celle de Bellefeuille. Au cœur de sa démarche se trouvent les mots qui inaugurent l'ouvrage : "[l'essai est] écrit par un homme et [...] se refuse à proposer une stratégie aux femmes en lutte; il combat pour elles, il ne peut dire leur

\footnotetext{
10 Bien que réduite à des indications schématiques dans cet article, l'évolution de l'avant-garde formaliste a fait l'objet d'analyses historiques, voire englobantes (Pelletier, 1986) ou, tout à l'opposé, de lectures pointues inspirées de la théorie du champ de Bourdieu (Milot, 1988). Le chapitre premier de mon mémoire, basé sur l'examen des poétiques d'un corpus revuiste, a montré que la poésie québécoise à la fin des années 1960, axée sur la forme comme l'a mis de l'avant le structuralisme, a progressivement pris des inclinaisons contre-culturelles avant d'opérer un virage plus politique (sémiologie et structuro-marxisme). Dans cette logique de générations du formalisme, le relais de l'avant-garde ou sa dissolution survient avec l'essoufflement des efforts militants des marxistesléninistes qui contrastent avec le retour à la lisibilité qu'opèrent un certain nombre d'écrivains. À cet égard, j'avance l'hypothèse que les courants poétiques associés au féminisme sont les derniers qui conservent un côté corrosif et engagé, ouvrant ainsi vers des questions culturelles de migration. Durant les décennies 1980-1990, les écritures migrantes et les problématiques corollaires (le pluriel, l'identitaire et l'hybride dans une perspective postmoderne et postcoloniale) tendent à annuler la logique des avant-gardes historiques (Bürger, 2013) ou à en être une ultime incarnation (Guay, 2015), puisqu'il s'agit des mêmes acteurs et qu'ils usent alors de stratégies rhétoriques "nouvelles » (patchwork, fragment, intertextes, recyclage, etc.) pour dire la diversité culturelle.
} 
combat: à elles de le dire, dans leurs propres termes» (Lascault, 1977, p. 5). Or, Corriveau précise dans ce qui pourrait s'apparenter à un appel : « [à] nous d'ajouter les fragments, de percevoir en nous-mêmes cette totalité féminine » (Corriveau, juin 1978, p. 72). Il s'adresse ici à une communauté d'hommes - un «nous » qui doit construire des représentations plurielles de la femme - invités à puiser en eux-mêmes pour faire surgir ces nouvelles images. Corriveau offre ainsi une configuration formelle inhabituelle du genre, un lieu de médiation, qui subvertit le dispositif de la différence des sexes.

Ce brouillage de la frontière entre féminin et masculin, qui détache et relativise les valeurs associées aux socles biologiques, n'est pas sans rappeler une perspective issue du queer (Butler, 2004; Delphy, 2001; Preciado, 2000; Wittig, 1992) postulant que le genre est prédiscursif, socialement construit et performé dans le langage. Chez Corriveau, cette notion même de mélange, de contamination, ce que Butler nomme le «gender trouble» (1990), s'oppose à la fixité des partitions identitaires. Il pointe ainsi son adhésion à la critique épistémologique, au «travail de révolte actuelle » (Corriveau, juin 1978, p. 73), de sape du langage caractéristique aux écrivaines de la $N B J$. Sa posture remet en question les représentations de la littérature féministe au Québec lorsqu'il dénonce le "phallocrate [qui] court ici le risque de reconnaître quelques-uns de ses objets, quelques-unes de ses pensées trop longtemps crues secrètes » (Corriveau, juin 1978, p. 73). Des mots loin d'être tendres, assez durs, desquels il s'exclut d'abord, avant de conclure que « [c]hose certaine, il faut tenir à ce petit livre [celui de Lascault], s'y reconnaître, si on ne peut naître ailleurs, au-delà, 
renouvelé à partir de lui » (Corriveau, juin 1978, p. 73-74). Son refus de la binarité des identités sexuelles et du genre est manifeste, et son désir de «naître ailleurs » annonce ce qu'Isabelle Boisclair et Lori Saint-Martin ont qualifié a posteriori de «troisième modèle, issu du féminisme et lié à la mouvance en matière de diversité sexuelle (queer) et postmoderne » $(2006, \text { p. 6) })^{11}$.

Pour ainsi dire, le prétexte de la recension de l'œuvre permet à Corriveau, tout comme à Bellefeuille, de tisser une solidarité intellectuelle avec les écrivaines déjà bien établies au sommaire de la $N B J$, où la poétique féministe a trouvé un lieu d'élaboration, qui cristallise ses principaux enjeux (Godard, 1983). Par leurs analyses littéraires, des métadiscours, ils alimentent, à leur manière, le nombre croissant de réflexions qui définissent l'intense période de radicalité de l'écriture des femmes au Québec, entre 1974 et 1979 (Boisclair, 2004, p. 158). Corriveau et Bellefeuille se font le relais d'œuvres qui offrent une lecture nouvelle de la société patriarcale et de ses fondements philosophiques. Certes, leurs textes critiques focalisent sur les femmes et le féminin, mais leur production poétique à la $N B J$ rend encore plus manifeste leur démarche. Pour y parvenir, ils signent des proses poétiques et des récits qui montrent aussi un retour à la lisibilité, et à l'écriture figurative, sans toutefois évacuer complètement la recherche formelle et l'intertextualité.

\footnotetext{
11 Ciblant son lien viscéral et sa venue à l'écriture, Corriveau dira, dans une entrevue accordée à $L a$ Presse : «[...] je suis né parce que les femmes ont écrit je suis devenu auteur parce qu'elles ont écrit et que je les ai lues et que ça sollicite chez moi une parole. Je me suis senti tellement interpellé par le discours féministe et les nouvelles écritures de femmes des années soixante-dix que j'ai compris là que j'avais à dire des choses d'une sexualité qui était masculine, avec tous les risques que ça pouvait comporter» (Côté, 1992, p. C1).
} 


\section{"Marquer la femme en soi » : poésie, récit et fiction théorique}

Dans les pages de la $N B J$, les contributions poétiques des hommes travaillent non seulement en continuité avec les visées féministes, mais elles tissent pour la plupart un circuit de références. En 1977, «Le texte infini » de Corriveau est dédié à sa compagne Louise Cotnoir, écrivaine avec qui il codirigera la NBJ (1981-84). Des références à Louise Dupré parsèment les textes de Bellefeuille (janvier 1982, p. 45), et Yolande Villemaire se voit aussi dédier plusieurs passages des poésies de Claude Beausoleil (avril 1978, p. 40). Les deux écrivains, Corriveau et Bellefeuille, qui renvoient souvent au travail de l'un et l'autre (Bellefeuille, 1976; Corriveau, 1977), partagent également avec Claude Beausoleil et André Beaudet, un panthéon des grands écrivains, plutôt des grandes «théoriciennes » ou «théoriciens » qui ont marqué leur pratique poétique. Fragment d'un discours amoureux de Roland Barthes (1977) est constamment sollicité pour penser l'amour en relation avec le langage, tout comme les écrits de Madeleine Gagnon, de Yolande Villemaire, de Nicole Brossard, de Josée Yvon et d'Hélène Cixous ${ }^{12}$. Qui plus est, Corriveau, Bellefeuille, Beausoleil et Beaudet ont recours aux mêmes procédés postmodernes pour déconstruire le «logocentrisme», comme le recyclage, le fragment, le collage et l'intertexte qu'ont ciblés les principales études sur la NBJ (Godard, 1983; Forsyth, 1983). Les pratiques

\footnotetext{
12 Cixous donne deux séminaires à l'Université de Montréal (hiver 1973, automne 1974) auxquels assistent Madeleine Gagnon, Philippe Haeck, Hugues Corriveau, Normand de Bellefeuille et Nicole Brossard. Comme le souligne Jean Larose, l'enseignement de Cixous eut beaucoup d'influence sur la génération $B J / N B J$ (1987, p. 158-159).
} 
de ce petit groupe d'écrivains masculins, d'où s'élève le spectre d'une posture collective, tentent de renverser la domination du patriarcat dans le langage.

En décembre 1980, «Acte délibéré », une prose poétique d'Hugues Corriveau, montre plus distinctement son appartenance au groupe d'écrivains «féministes » de la NBJ. Ce dernier écrit :

[d]ans les parages des femmes, en ce moment, beaucoup de paroles qui me font m'inquiéter de la mienne [...] Ainsi les hommes se dévêtent sur leurs machines. [...] Aussi, leur faut-il imaginer d'autres façons de presser, de tenir en laisse, de savoir à qui les coups servent. Mais là, ils n'ont pas compris (Corriveau, janvier 1980, p. 51).

Corriveau pointe ici les «hommes» avec distance; le pronom «ils» au pluriel sert d'élément de distinction. Tout en contraste avec Bellefeuille, ce « nous » ne représente pas un groupe d'écrivains capable de solidarité et d'ouverture pour dire ce qui relèverait de la féminité. Les « hommes » que cible Corriveau «n'ont pas compris ».

Mêlant fiction et théorie, Corriveau donne à lire les termes peau, jambes, cuisses, visages des femmes, ventre, bassin, épiderme, doigt, sang, cycle des menstruations, ongles et corps comme autant d'expressions qui caractérisent la métaphore du «cortex», orientée vers les recherches féministes. Son écriture est désirante et énergétique, rappelant la pratique brossardienne, par les syntagmes plaisir, sexe, peaux mouillées, chaleur, hommes bandés, jus, fentes, désirs, nudités, débordements liquides, subites énergies, défloraison renouvelée, odeur de menstrues et impression intime. La métaphore du corps-texte se conjugue ici avec le «plaisir du texte» (Barthes, 1973) que l'on retrouve abondamment chez les poètes formalistes 
ou contre-culturels, notamment à la revue $C u l Q$, mais en vouant du respect et de l'admiration aux femmes.

Corriveau renforce davantage ce nouage lorsqu'il énonce: «[m]ais ma peau, quant à elle, parfois si féminine, et ses désirs. Du trouble : un corps et des nudités. Y voir absolument. Mais plutôt, dans le recours aux lieux de toutes sortes, on ne saisit pas autrement l'une et l'autre» (Corriveau, janvier 1980, p. 54). Sa prose poétique offre une perspective privilégiée sur les rapports entre hommes et femmes. En outre, l'écrivain remet en cause l'hétéronormativité et les modèles identitaires dualistes lorsqu'il cherche à « [m]arquer la femme en soi » (Corriveau, janvier 1980, p. 56), une performativité du genre qu'il pousse encore plus loin.

Ne pas connaître la sécrétion multipliée qui parle de soi. Se chercher, aussi, dans tout ce débordement pour s'en permettre d'autres, dans sa propre absence, sans saisir tout à fait ce qui ne va pas. Faire de son érection une autre valeur, sans aucun doute. Spectaculaire, sans doute. Mais sans pouvoir, ici, dans sa déconcertante insignifiance (Corriveau, janvier 1980, p. 57).

Corriveau attaque des symboles forts de la « puissance » masculine, soit l'érection et le phallus qu'il réduit au vide de sens, à l'«insignifiance ». En parallèle, l'écrivain construit plutôt l'image d'un homme à l'écoute de ses désirs, cherchant « la femme en soi ». Cette posture est inédite non seulement à l'égard de la tradition littéraire québécoise, mais surtout vis-à-vis des structures patriarcales et oppressantes du langage dénoncées par les femmes à la même époque. En somme, Corriveau opère un 
renversement des antagonismes, très peu étudié, à ma connaissance, dans l'histoire de la littérature québécoise ${ }^{13}$.

À cet effet, je voudrais maintenant analyser un court récit d'Hugues Corriveau qui fit l'objet d'un numéro complet des herbes rouges, sous le titre « Du masculin au singulier » (1981). Bien que ce texte narratif déborde du corpus de La Nouvelle Barre $d u$ jour, il entre en dialogue avec les essais et la poésie précédemment étudiés et mérite en cela une attention particulière. Comme son titre l'indique, il s'agit d'un récit de soi, écrit à la première personne du singulier, centré sur un individu et ses désirs subjectifs. Un homme, le personnage principal et moteur de la narration, y décline ses envies sexuelles autant que ses peurs et ses limites, dans ce qui s'apparente à un journal introspectif de sa relation amoureuse et sexuelle avec une inconnue. L'incipit place déjà le décor: une femme, un lit, une chambre, la peau mate des jambes, des tissus, de l'inattendu et la lumière du matin. Le narrateur dépeint ainsi la scène.

J'en sentirais quelque parenté. Mais l'étrangère, celle-là qui ne se nomme pas, dans ses infirmités affichées, m'inquiète très heureusement [...] Il n'y a de femme en elle selon aucun modèle. Elle est ce qui ne s'accorde pas. Ni schème ni cela qui s'apprend. Et dans son reste de désordre, de résistance, je ne m'active pas. J'ai dans les yeux, tout au travers posées, des loupes, des lentilles qui, sans déformer, ne précisent pas d'identités. Elle s'appelle plusieurs et n'a pas de galbe. Elle s'étend dans ce lit, le mien, me happe sans attendre, indiscrète. (Corriveau, 1981a, p. 4-5)

Une tension érotique émane de cet extrait. Selon les impressions saccadées du narrateur, l'image et le corps de l'étrangère défient toutes normes et, à la fois, rassemblent les traits de plusieurs femmes. L'inaction contraste avec les minutieuses

\footnotetext{
${ }^{13}$ Les textes du romancier Jacques Poulin ont fait l'objet de quelques études cernant la construction du
} genre et de l'identité sexuée, mais les écrits en revue n'ont pas été abondamment sollicités. 
observations traduisant un désir et une curiosité envers cette partenaire anonyme, qui semble pourtant familière. Le regard qu'il porte ne cherche pas à déformer ses infirmités - l'amante est «mutilée» (Corriveau, 1981a, p.14), a une «jambe insoumise et inactive» (p. 29-30) - mais suscite en lui de la stupéfaction (p.6). Corriveau met ici en évidence une féminité anormale qui contraste avec les traits construits et véhiculés par la société et les médias, et par les représentations et les standards de la corporéité circulant dans l'espace social, qui produisent des « filles en série » (Delvaux, 2013).

Dispositif sémiotique de résistance depuis les débuts de l'activisme féministe (Bard, 2014), le corps féminin se présente sous le signe de la différence. Le narrateur revient sans cesse à son brûlant désir d'étreindre et d'aimer cette femme mystérieuse « proprement indécente dans ce qu'elle est amputée » (Corriveau, 1981a, p. 11). Son corps féminin constitue le siège d'un imaginaire autre que celui des canons. Étourdissante, elle le captive, s'enhardit jusqu'à le fixer du regard, montrant des similitudes avec les figures fortes de son enfance grâce à l'audace et la franchise dont elle fait preuve. "[D]evant l'évidence de sa beauté» (Corriveau, 1981a, p.33), l'homme s'épanche sur ses envies : «quand je la vois, je me sens pour elle quelques fantasmes d'amoureux» (p. 35). Par le biais de son personnage, l'auteur décape les modèles culturels dominants - il fait montre d'une subjectivité et d'une sexualité plus authentiques - recadrant ainsi de nouveaux possibles identitaires. 
Si Corriveau cherche la féminité en lui-même et perçoit, dans sa poésie, le genre dans sa pluralité, son récit instaure plutôt un espace de déstabilisation qui met l'accent sur la fragilité et la sensibilité masculines. Fasciné par le corps atypique de sa maîtresse, le personnage ne peut nier son attirance sexuelle, mais peine à entrer en contact. Il raconte :

[m]ais elle n'est pas celle qu'on nous a enseignée. J'ai alors, bras ballant, des pensées tristes devant les inutiles apprentissages qui ne m'y ont pas préparé. Pourtant, dans ce qui se laisse, de la métamorphose, près d'une évocation chrysalide, même du masque avant d'être. Je n'ai rien dans les mains que mes organes mous, réchauffés, tendrement subtils (Corriveau, 1981a, p. 5).

Derrière le «nous» se dissimule une série de critiques portant sur l'inanité des connaissances. Pour pallier cette absence, l'expérience en elle-même est mise en valeur. Dès lors, la sexualité apparaît hors des injonctions de performance; ses «organes mous, réchauffés » conservent candeur et tendresse, signes d'une dissidence pratiquement assumée. Selon cette perspective, le narrateur est en décalage avec la masculinité hégémonique, fondée sur la virilité et le phallus (Connell, 2005 [1995]). En effet, ni pénétration ni coït ne surviennent au fil des pages; on y décrit surtout des caresses. Bien qu'il s'inscrive dans un cadre hétéronormatif, le récit offre une gamme de subjectivités et de sensations érotiques qui s'éloignent, sur le plan des pratiques sexuelles, du monde viril et du pouvoir masculin. La relation sexuelle avec l'étrangère permet de penser les rapports de sexe et de domination, la sexualité et l'identité masculines sous un nouvel éclairage. 
$\mathrm{Au}$ travers des confessions intimes se font entendre sans cesse des remarques amères. L'impuissance de l'homme devient un prétexte à critiquer les représentations de la masculinité :

[c]ar même là, ça guette de partout les élégances trop prononcées qui ne sont pas suffisamment mâles, qui ne tiennent pas assez compte des exigences de l'heure. Au milieu de ses gestes, même, il faut reconnaître. C'est cela surtout, dans les mauves imprécis des cigarettes et des alcools, qu'elle dit. Quelqu'une remarque mon malaise, et il est si difficile de s'associer. Elle voit, dans les strass et les anthracites du siècle dernier, comme un monstre inapte, mal assuré (Corriveau, 1981a., p. 8).

Se sentant surveillé, le personnage dénonce les comportements prescrits par le modèle hégémonique. Il revient ainsi sur l'image de l'homme efféminé qu'on cherche à débusquer, faute de l'assumer. Bien que l'inconnue lui fasse reconnaître la sensibilité qui l'habite, un malaise l'envahit et traduit à nouveau son incapacité. Ces critiques ciblent les rapports de pouvoir existant entre les genres, plus précisément au sein de chacun des genres. La domination s'exerce non seulement sur les femmes, mais aussi sur les masculinités subordonnées, créant une hégémonie interne qui subvertit l'idée même d'une masculinité unifiée et cohérente. Aux prises avec «[s]es ignorances légendaires d'homme » (Corriveau, 1981a, p. 14), « [s]es incertitudes [et s]es maladresses » (p. 35), le personnage avoue : « je n'ai pas de parole d'homme pour mes propres infirmités » (p. 11). En ciblant ses lacunes et, en général, l'inaptitude des hommes à entrer en relation avec les femmes, il réitère : « [j]'affirme mon ignorance et l'imprécision hagarde de mes semblables» (p. 17). Au final, « Du masculin singulier» se fonde sur le refus des pratiques sexuelles et sociales, qui assurent la reproduction du patriarcat, et s'inscrit dans une volonté de remodélisation du 
masculin par les hommes eux-mêmes. Intègre, le personnage résiste aux injonctions de la masculinité hégémonique, «au prétentieux décorum des mâles » (Corriveau, 1981a, p. 21), en acceptant ses désirs enfouis et la mollesse de son sexe; des actes qui disent paradoxalement sa puissance d'agir, son agentivité.

Si les écrits de Corriveau à la $N B J$ et aux herbes rouges montrent un travail d'affirmation, les fictions théoriques engagent une démarche tournée vers le pragmatisme en ce qu'elles intègrent une dimension métalangagière ou métadiscursive. Sous le titre « Masculinarités » (1977), Joseph Bonenfant publie un texte en prose, autoréflexif, qu'il adresse à une inconnue représentée par le pronom « elle ». Il fait référence au mythe de la création de la femme à partir de la cote de l'homme. Ce retour à la genèse lui permet de jouer des concepts psychanalytiques :

[1]a phalle à Freud se mettant de la partie, mettant la main sur la partie, émet ceci que ça, c'est moi, l'homme. Désirez, réintégrez, comment mettrez-vous le pied dans le symbole, vous qui n'avez que des seins-bols. Entre mon pêne de père et ma phalle de crassie, je me suis symbolisé à la masse culine; restez derrière la porte avec votre peine de mère et votre phalle sein-bolisée et attendez que le temps passe (Bonenfant, décembre 1977, p. 107).

On remarque ici l'utilisation d'un procédé caractéristique de la poésie formaliste, et dans une certaine mesure, de la poésie féministe : les transformations de mots par métaplasme. Ces altérations phonétiques s'attaquent au logocentrisme en minant l'intégrité des mots par adjonction, suppression ou déplacement de phonèmes ou de lettres. Dans l'extrait de Bonenfant, le mot symbole devient « seins-bols», phallocratie (comme système qui assure et justifie la domination des hommes sur les femmes) est décomposé en trois termes, "phalle de crassie», qui associent l'organe sexuel masculin (phallus) à un membre grossier ou sale dont on n'arrive pas à se défaire 
(crasse); un hiatus disant la brisure et l'ironie. Liée à sa forme réflexive, la fiction théorique aborde ainsi des points de langage ou d'expression pour dénoncer des emplois sexistes ou ridiculiser des motifs de l'identité masculine. Plus précisément, ces procédés poststructuralistes font violence au langage et critiquent les représentations des hommes qui sont nommés, chez Bonenfant, les «masculinaires » (Bonenfant, 1977, p. 107), en écho à « tortionnaire ».

En 1980, Normand de Bellefeuille opère un renversement de la vision de l'homme comme bourreau des femmes. Sa poétique puise au canevas de la lettre, genre épistolaire qu'empruntent souvent les féministes des années 1970-80. Bellefeuille écrit :

[c]hère amie, je vous dépêche ma différence sexuelle sous petit pli afin que, dans la conversation et la diction des monstres, question émouvante, un peu de parole atroce et douloureuse s'installe, suppliante, je sais déjà que quelques figures arriveront à évoquer distinctement le discours délicat des monstres; disons-le pour l'instant " suffisant à l'amour » et urbain, faisons du deux à la façon du politique et de l'étreinte. Croyez-moi bien que mes intentions sont simples, saines et que la machine, bien que souveraine... (Bellefeuille, juin 1980, p. 111).

Cet extrait, dont les intertextes réfèrent aux concepts de «machines désirantes » de Deleuze et Guattari dans L'Anti-OEdipe (1972), montre l'émergence d'une figure de l'amant attentionné, de l'ami et même du complice chez Normand de Bellefeuille14. Ce recours aux «machines désirantes » pour dire la complicité est tout à fait novateur. Chez Deleuze et Guattari, la notion renvoie au multiple et procède d'une logique du désir qui ne borne pas le genre au féminin ou au masculin (réduit à des unités), mais

${ }^{14}$ En 1981, Hugues Corriveau reprend aussi la figure du complice lorsqu'il décrit les prouesses de sa compagne écrivaine : " [s]es habiletés d'écriture, ses grandes pages toutes pleines de mots et de la voix. [...] Par hasard, cela fait de l'élégance dans le mouvement. Cela s'ouvre tout juste pour que je vois [sic] complice l'indélicatesse de certaines sueurs » (mars 1981, p. 44). 
plutôt à des agencements infinis, des entités rhizomatiques. Bellefeuille propose donc un modèle conceptuel basé sur des rapports de sexe qui confrontent les stéréotypes sexistes et les schèmes figés vers l'expérimentation d'identités sexuelles composites, transitoires et moins univoques.

Au final, les écrits de Bellefeuille et Corriveau manifestent un désir d'adhésion, un mouvement de solidarité avec les écrivaines de l'avant-garde féministe qui publient à $N B J$. Dans un certain retour à la lisibilité, ces contributions font appel à des intertextes communs pour dire le corps-texte, la féminité, le masculin et le désir sous un angle inédit. Il faut, à mon avis, dépasser le constat de Roger Chamberland en 1992, affirmant que «dorénavant, il sera difficile d'écrire sans tenir compte de cette écriture-femme dont l'intensité et l'émotion auront des répercussions même parmi les textes de nombreux poètes masculins » (p. 286). En effet, comme j'ai tenté de le montrer, les textes de ces auteurs à la $N B J$ ont fait surgir des représentations renouvelées de l'homme (l'amant, le complice, l'ami), non pas en réponse aux féministes, mais dans l'idée commune de dire, de nommer, de vivre et d'afficher leur appartenance à la sphère littéraire. Ils ont affirmé plus clairement l'idée d'un groupe mixte lié au féminisme, qui a ouvert la voie à la constitution d'un imaginaire non binaire des sexes, du genre et de l'identité, dont les assonances avec la réflexion contemporaine sur la diversité et la pensée queer sont surprenantes. À l'aune des poétiques masculines étudiées ici, qu'un examen des correspondances et des réseaux permettrait certainement d'approfondir, La Nouvelle Barre du jour doit être appréhendée comme un lieu de prise de parole et d'écriture pluriel et hétérogène. Il 
apparaît juste d'affirmer à cet égard que l'avant-garde féministe, souvent basée sur une différenciation sexuelle pensée en fonction du génital, a pu compter sur la collaboration d'auteurs pour s'engager dans un processus de renouvellement des formes et des pratiques poétiques au seuil du postmodernisme.

\section{Bibliographie}

\section{Corpus primaire}

Beausoleil, Claude et André Roy (1974), "Pour une théorie fictive », Cul Q, nos 4-5, p. 39-50.

BEAuSOLEIL, Claude (1977), « Le pays le texte et après », Hobo-Québec, nº 32, p. 19.

BeAusoleIL, Claude (avril 1978), « Déplacements \& failles ou Bruits d'actes suivi de Les vagues blanches », La Nouvelle Barre du jour, no 65, p. 40-53.

Bellefeuille, Normand de (1976), «Le texte justement », les herbes rouges, n 34, n. p.

Bellefeuille, Normand de (septembre 1977), «Le corps mineur ou l'impossible lyrisme », La Nouvelle Barre du jour, n 58, p. 82-91.

BELlEFEUILlE, Normand de (novembre 1977), « La gageure du lisible [compte rendu de Serge Gauthier, Glottes, les herbes rouges, n 53, juillet 1977] », La Nouvelle Barre du jour, $\mathrm{n}^{0} 60$, p. 70-71.

Bellefeuille, Normand de (juin 1980), «Dans la conversation et la diction des monstres (extrait) », La Nouvelle Barre du jour, nos 92-93, p. 109-113.

Bellefeuille, Normand de (janvier 1982), « Le livre du devoir (extraits) », La Nouvelle Barre du jour, no 109, p. 43-48.

Bonenfant, Joseph (décembre 1977), «Masculinarités », La Nouvelle Barre du jour, $\mathrm{n}^{\circ} 61$, p. 104-115.

Brossard, Nicole (1973), « Vaseline », La Barre du jour, no 42, p. 11-17. 
ColETTE, Jean-Yves (novembre 1977), «Une vie prématurée (extrait)», La Nouvelle Barre du jour, no 60, p. 19-29.

CoRriveau, Hugues (décembre 1977), «Le texte infini », La Nouvelle Barre du jour, $\mathrm{n}^{\circ} 61$, p. $5-18$.

CoRriveau, Hugues (juin 1978), «La féminité en question! [Compte rendu de Gilbert Lascault, Figurées, défigurées. Petit vocabulaire de la féminité représentée, Paris, UGE, coll. « 10/18», 1977, 222 p.] », La Nouvelle Barre du jour, nº 67, p. 71-74.

CoRriveau, Hugues (janvier 1980), «Acte délibéré », La Nouvelle Barre du jour, nº 86, p. 50-57.

CoRriveau, Hugues (mars 1981), "Travaux de sape », La Nouvelle Barre du jour, $\mathrm{n}^{\text {os }} 100-101$, p. $43-46$.

CoRRIVEAU, Hugues (1981a), « Du masculin singulier », les herbes rouges, n 86, p. 3-36.

Estuaire (mai, août 1977), «[Bulletin d'abonnement] », La Barre du jour, nos 56-57, p. 262.

FRÉMONT, Gabrielle (1985), "V.3 Le féminisme à la NBJ : un second souffle », Voix et images, vol. 10, n² 2, p. 133-137, id.erudit.org/iderudit/013878ar.

GAY, Michel (janvier 1978), «Un nerf d'aller », La Nouvelle Barre du jour, no 62, p. 4150.

LASCAUlt, Gilbert (1977), Figurées, défigurées. Petit vocabulaire de la féminité représentée, Paris, UGE, coll. «10/18».

Y.M. [Yvan Mornard]/Quoi (1967), « Le laboratoire », Quoi, vol. 1, no 2, p. 3-6.

\section{Corpus secondaire}

ANDRÈs, Bernard (1986), «Institution et avant-garde : Herbes rouges versus Nouvelle Barre du jour», dans Maurice Lemire (dir.), L'institution littéraire, Québec, CRELIQ/IQRC, p. 105-116.

BARD, Christine (2014), « "Mon corps est une arme", des suffragettes aux Femen », Les Temps Modernes, no 678, p. 213-240.

BARTHES, Roland (1973), Le plaisir du texte, Paris, Seuil. 
Beaudry, Jacques (dir.) (1998), Le rébus des revues. Petites revues et vie littéraire au Québec, Québec, Presses de l'Université Laval.

BERgeron, Marie-Andrée (2013), "NOUS AVONS VOULU PARLER DE NOUS». Le discours éditorial des féministes québécoises (1972-1987) dans Québécoises deboutte!, Les têtes de pioche et La Vie en rose, thèse de doctorat, Québec, Université Laval.

BoISClair, Isabelle et Lori SAINT-MARTIN (2006), "Les conceptions de l'identité sexuelle, le postmodernisme et les textes littéraires », Recherches féministes, vol. 19, $\mathrm{n}^{\circ} 2$, p. 5-27, id.erudit.org/iderudit/014841ar.

BoISCLAIR, Isabelle (2004), Ouvrir la voie/x. Le processus constitutif d'un sous-champ littéraire féministe au Québec (1960-1990), Québec, Nota bene.

Biron, Michel, François Dumont et Élisabeth NARdout-LAFARGE (2010 [2007]), Histoire de la littérature québécoise, Montréal, Boréal, coll. « Boréal compact ».

BÜRGER, Peter (2013 [1974]), Théorie de l'avant-garde, Paris, Éditions Questions Théoriques.

ButLER, Judith (1990), Gender Trouble: Feminism and the Subversion of Identity, New York/Londres, Routledge.

ButLER, Judith (2004), Undoing Gender, New York/Londres, Routledge.

Chamberland, Roger (1992), «Les voies/voix multiples de la poésie québécoise contemporaine », Recherches sociographiques, vol.33, $\mathrm{n}^{\circ} 2$, p. 277-298, id.erudit.org/iderudit/056694ar.

Connell, Raewyn W., (2005 [1995]), Masculinités. Enjeux sociaux de l'hégémonie, Paris, Éditions Amsterdam.

CôtÉ, Lucie (1992), «Les p’tits oiseaux, les fleurs, l’amour, éros... », La Presse, 29 mars, p. C1 et C2.

Deleuze, Gilles et Félix GuatTari (1972), Capitalisme et schizophrénie. 1 : L’Anti-Oedipe, Paris, Les Éditions de Minuit.

DelPhy, Christine (2013 [2001]), L’ennemi principal. 2. Penser le genre, Paris, Éditions Syllepse, coll. « Nouvelles Questions Féministes ».

DelvauX, Martine (2013), Les filles en série, Montréal, Éditions du remue-ménage. 
Dion, Robert (1994), "L'analyse littéraire à la $B J / N B J$ : traditions de lecture et modernité québécoise», dans Joseph Melançon, Nicole Fortin et Georges Desmeules (dir.), La lecture et ses traditions, Québec, Nuit blanche éditeur, p. 213231.

Dupré, Louise (1989), Stratégies du vertige - trois poètes, Nicole Brossard, Madeleine Gagnon, France Théoret, Montréal, Éditions du remue-ménage.

FoRSYTH, Louise (1983), «Les numéros spéciaux de La (Nouvelle) Barre du jour. Lieux communs, lieux en recherche, lieu de rencontre», dans Suzanne Lamy et Irène Pagès (dir.), Féminité, subversion, écriture, Montréal, Éditions du remue-ménage, p. 175-184.

FORTIN, Andrée (1990), "Les intellectuels à travers leurs revues», Recherches sociographiques, vol. $31, \mathrm{n}^{\circ} 2$, p. 169-200.

GODARD, Barbara (1983), "La Barre du jour: vers une poétique féministe », dans Suzanne Lamy et Irène Pagès (dir.), Féminité, subversion, écriture, Montréal, Éditions du remue-ménage, p. 195-205.

GuAY, Élyse (2015), La revue Dérives (1975-1987) et l'écriture migrante: introduire le Tiers dans la littérature québécoise, mémoire de maîtrise, Montréal, Université du Québec à Montréal.

LAROSE, Jean (1987), La petite noirceur, Montréal, Boréal, coll. « Papiers collés ».

NEPVEU, Pierre (1981), "Petites misères du masculin singulier », Lettres québécoises, no 22, p. 29-31.

NEPVEu, Pierre (1985), «BJ/NBJ : difficile modernité », Voix et images, vol. 10, n 2, p. 159-165, id.erudit.org/iderudit/013881ar.

NePveu, Pierre (1988), L'écologie du réel. Mort et naissance de la littérature québécoise contemporaine, Montréal, Boréal, coll. « Papiers collés ».

Milot, Pierre (1988), La camera obscura du postmodernisme, Montréal, l'Hexagone, coll. « Essais littéraires ».

PelLETIER, Jacques (dir.) (1986), L'avant-garde culturelle et littéraire des années 70 au Québec, Montréal, Université du Québec à Montréal, Les Cahiers du département d'études littéraires, $n^{0} 5$.

Pluet-Despatin, Jacqueline (1992), «Une contribution à l'histoire des intellectuels : les revues », Les Cahiers de l'IHTP, no 20, p. 125-136. 
Preciado, Paul B. (2000), Manifeste contra-sexuel, Paris, Balland.

WiTTIG, Monique (1992), The Straight Mind, Boston, Beacon Press.

\title{
Résumé
}

Le présent article s'attache à relire les contributions d'auteurs masculins à $L a$ Nouvelle Barre du jour, revue érigée en lieu d'émergence de l'avant-garde poétique féministe par la critique et par les pairs. Au seuil des années 1980, Hugues Corriveau et Normand de Bellefeuille y affirment l'idée d'un groupe mixte qui ouvre la voie à la constitution d'un imaginaire non binaire des sexes, du genre et de l'identité. Leur écriture désirante et énergétique rappelle la pratique brossardienne et participe au renouvellement discursif de la revue, entrant en résonnance avec la réflexion contemporaine sur la diversité et la pensée queer.

\begin{abstract}
The following article's goal is to read through the contributions of male writers to the Nouvelle Barre du jour, a journal that brought in light feminist avant-garde poetry. By the end of the 1970s, Hugues Corriveau and Normand de Bellefeuille assert in their texts a new vision that abolishes distinction between sex as biological facticity, and gender binary. Their desiring and energetic writing, similar to Nicole Brossard's work, contributes to the renewal of the journal and echoes today's queer theories.
\end{abstract}

